\title{
Identification of a Novel Missense Mutation in the Fibroblast Growth Factor 5 Gene Associated with Longhair in the Maine Coon Cat
}

Lisa G Shaffer ( $\square$ lshaffer@pawprintgenetics.com )

Genetic Veterinary Sciences, Inc https://orcid.org/0000-0002-2848-8103

Griffin D Shaffer

Genetic Veterinary Sciences Inc

Blake C Ballif

Genetic Veterinary Sciences Inc

Kathryn Meurs

North Carolina State University College of Veterinary Medicine

Helen Flores-Smith

Genetic Veterinary Sciences Inc

Research Article

Keywords: Cat, FGF5, longhair, Maine Coon, Felis Catus

Posted Date: June 4th, 2021

DOl: https://doi.org/10.21203/rs.3.rs-575619/v1

License: (9) This work is licensed under a Creative Commons Attribution 4.0 International License.

Read Full License 


\section{Abstract}

Hair length can be a highly variable trait within the Felis catus species, varying between and within different cat breeds. Previous research has demonstrated this variability is due to recessive mutations within the fibroblast growth factor 5 (FGF5) gene. Following a clinical genetic screen, four long-haired Maine Coons were identified that had only one copy of a known FGF5 mutation. We performed DNA sequencing on two of the Maine Coons and identified a missense mutation in FGF5 c.G>A625 p.A193T (Mutation 5, M5). Genetic screening via restriction digest was then performed on an additional 275 cats of various breeds, revealing two more Maine Coons heterozygous for M5 and a previously known mutation. No additional cases were identified after in silico analysis of 68 whole genome cat sequences from various breeds, demonstrating that this novel mutation is most likely a breed-specific variant for the Maine Coon, contributing to the longhair phenotype in about $3 \%$ of cats.

\section{Introduction}

The fibroblast growth factor 5 (FGF5) protein encoded by the FGF5 gene mediates the mammalian hair follicle cycles through the periods of growth, involution, and rest, resulting in various hair lengths between and among species due to variants within the FGF5 gene (Housley and Venta, 2006). Demonstrated in both dogs (Dierks et al 2013) and cats (Drögemüller et al 2007; Kehler et al 2007), longhair is recessive to shorthair and requires homozygosity or compound heterozygosity of variants in FGF5.

Specifically in cats, the impact of FGF5 on hair length has been demonstrated within different breeds, showing how variants of FGF5 cause longhair (Drögemüller et al 2007). Four variants have been identified: Mutation 1 (M1), is an insertion of a thymine base found in the Ragdoll breed; Mutation 2 (M2), is a cytosine to thymine conversion found in the Norwegian Forest Cat breed; Mutation 3 (M3), is a deletion of a thymine base found in Ragdoll and Maine Coon breeds; and Mutation 4 (M4), is an adenine to cytosine conversion found in most longhair cat breeds (Drögemüller et al 2007, Kehler et al 2007). All four variants can be found in a homozygous state or a compound heterozygous state involving multiple variants (Kehler et al 2007).

We have a developed a genetic screen for cats that tests 85 different variants causing diseases and traits (www.mycatscan.com). Among the trait tests are the four known variants in FGF5 for longhair (M1-M4). Genetic screening of more than 950 cats identified 4 Maine coons with only one of the known variants in FGF5, yet exhibiting longhair. Sequencing of FGF5 in two of these identified Maine Coons, with additional screening in 144 Maine coon cats and confirmation in relatives, led to the identification of a fifth, novel variant associated with longhair in the Maine coon.

\section{Materials And Methods}

\section{Study Cohort}


All cats in the study were privately owned and samples were collected by the cats' owners.

PERFORMAgene PG-100 (DNA Genotek, Ontario, Canada) buccal swabs were used to collect cheek cells from cats. DNA was extracted using the KingFisher Flex Purification System (ThermoFisher Scientific, MA, USA) as specified by the manufacturer. DNA concentrations were quantified with a NanoDrop 2000c spectrophotometer (ThermoFisher Scientific), and samples over $40 \mathrm{ng} / \mathrm{uL}$ were diluted in DNA suspension buffer to $20 \mathrm{ng} / \mathrm{uL}$. Samples were genotyped using the CatScan of 85 known variants associated with diseases and traits using an Agena Biosciences MassArray with the iPLEX Gold PCR kit and SpectroCHIP kit-CPM (Agena Biosciences, San Diego, CA, USA) with methods as previously described (Adams et al. 2003; Maksymowych et al. 2003). Among these, 277 cats of various breeds were selected for further study (Table 1). Maine Coons that were found to only have one copy of the known variants were confirmed to be longhair via personal communication with the owners and pictures when possible. Owners were also contacted for related cat samples, preferably tom and queen or full siblings when available, to determine the mode of inheritance.

\section{Sequencing}

Sequencing was performed using the ThermoFisher SeqStudio Genetic Analyzer. Four primers were designed with M13 adapters to cover the exons and intronic boundaries of FGF5 (See Table 2). PCR was prepared using the BigDye Terminator v3.1 Sequencing Kit (ThermoFisher Scientific) and purified using the BigDye XTerminator Purification Kit (ThermoFisher Scientific) then run on the SeqStudio Genetic Analyzer using the MediumSeq Sequencing Run Module. Sequence runs were analyzed manually using Chromas (Technelysium, Brisbane, Australia) and after the variant was identified, the sequence was BLATed to the ICGSC Felis_Catus_8.0 Assembly (UCSC Genome Browser) to confirm the variant was not a known benign polymorphism.

\section{Variant Screening}

Using the primer pair FGF5_5 (Table 2), a screening assay was developed via restriction enzyme digestion. PCR was setup using the ZymoTaq PreMix (Zymo Research, Irvine, CA, USA) and amplified with standard PCR conditions (40 cycles with the annealing temperature of $60 \mathrm{C}$ ). The restriction enzyme (Sau96I) was selected using NEBcutter V2.0 and sourced from New England BioLabs (NEB, Ipswich, MA, USA). Sau96I (G^GNCC) was used to digest the samples using standard conditions set by NEB. The resulting digest product was diluted 2:15 with PCR certified water and run on a 48 Well $2 \%$ Agarose E-Gel (ThermoFisher Scientific). E-Gels were visualized on the Analytik Jena UVP Transilluminator (Jena, Germany) and scored manually based on digest band sizes and intensity.

The genomes of 68 previously sequenced cats of various breeds (North Carolina State College of Veterinary Medicine) were compared via sequence alignment to the FGF5 DNA sequences obtained from the two test cats.

\section{Results}


To date, more than 950 cats have been tested using the CatScan panel of 85 known variants associated with diseases and traits in cats (www.mycatscan.com). Of these, four Maine coons, which are typically longhaired cats, were identified to be heterozygous for only one of the known variants in the FGF5 gene. The samples from two of these cats were used for sequencing of the FGF5 gene or genotyped for the novel FGF5 variant, with results as described below. An additional 275 cats of various breeds, including 144 Maine coons, were screened via restriction enzyme digestion on a $2 \% \mathrm{E}-\mathrm{Gel}$ and 68 cats were screened via in silico sequence alignment for a total of 345 different cats included in this study (Table 2). Samples represented 32 different breeds of cats of varying hair lengths: 3 hairless breeds, 15 shorthaired breeds, 7 longhaired breeds, and 8 breeds that can have either short or longhair.

\section{Sequencing}

We used samples from two Maine coon cats heterozygous for one known mutation and a control cat that is a compound heterozygote for M3 and M4 to search for additional variants within the FGF5 gene. Based on a recessive mode of inheritance, it was hypothesized that cats with longhair having only one copy of a known mutation were likely compound heterozygotes for a different variant. Through sequencing, the known variants were confirmed in each cat. Furthermore, a single, novel missense variant was identified c.G>A625 in the FGF5 sequencing (Fig. 1). The sequence data was submitted to NCBI GenBank with the accession number MZ311544. Figure 2 shows the location of this novel variant, M5, as compared to the four previously known variants. This variant causes an amino acid substitution of alanine to threonine at amino acid position 193 (p.A193T). PolyPhen predicts this mutation to be deleterious with a score of 1 (PolyPhen). The amino acid sequence was also compared against other species and showed that the region is highly conserved among various species (Fig. 3). No other variants were identified.

\section{Genetic Screen}

A PCR assay was developed to screen cats for this mutation to correlate its occurence with longhair and to determine if the variant was associated with any of the known longhair mutations (in cis), as known longhair mutations were already known in each of the cats. The screening was performed using a Sau96I restriction digest, with the wildtype sequence resulting in a $350 \mathrm{bp}$ and 400bp digested product and M5 sequence resulting in a 600bp (uncut) product. Cats with an M5 allele and one other known variant, showed the 350bp, 400bp and 600 bp products (Fig. 4). A total of 277 cats were screened using this assay. No shorthair or hairless cats were found to have this variant and no longhaired cats with two known mutations were found to have this variant. Two additional Maine Coons, who were not used for the sequencing, were found to be heterozygous for M5, for a total of four Maine Coons with one known mutation and the new M5 variant: M3/M5 ( $=2)$, M4/M5 ( $=2)$. Of the 68 additional cat sequences from various breeds analyzed in silico, none were found to have the M5 variant. No Maine coons were among those sequences analyzed.

\section{Inheritance}


After finding the variant in four unrelated Maine Coons, the owners were contacted and we requested buccal swabs from related cats, preferably parents or offspring. We received sample from three offspring of one Maine Coon who has the M5 variant (Fig. 5A) and received samples from the mother of a second Maine Coon with M5 (Fig. 5B). Each related Maine Coon was tested for M5 using the PCR screening assay and tested for the four known variants with the CatScan. All three offspring (Fig. 5A) and the mother in the second pedigree (Fig. 5B) were found to have the M5 variant, consistent with a recessive mode of inheritance.

\section{Discussion}

FGF5 is a known regulator of mammalian hair growth (reviewed in: Housley and Venta, 2006). Recessive mutations in mice contribute to the angora phenotype (Hébert et al. 1994), with variants in FGF5 contributing to longhair in dogs (Dierks et al. 2013) and other mammals (Legrand et al. 2014; Yoshizawa et al. 2015; Daverio et al. 2017; Yu et al. 2018; Zhang et al. 2019). Interestingly, in humans, molecular characterization of trichomegaly in consanguineous families identified recessive variants in FGF5 (Higgins et al. 2014).

The domestic cat is defined by a number of different breeds based on morphological appearance, including the presence of short hair or longhaired varieties (Alhaddad et al. 2021). Selective breeding for desired characteristics will fix certain phenotypes within a breed. Previously, four variants were described in the FGF5 gene that contribute to longhair in cats (Drögemüller et al 2007; Kehler et al 2007). We describe here a novel recessive allele in the FGF5 gene associated with longhair in the Maine Coon, following previous nomenclature, called Mutation 5, M5. This c.G>A625 results in an alanine to threonine substitution and is found to exist with known M3 or M4 FGF5 mutations in this breed as compound heterozygotes with phenotypically longhair. The alanine at position 193 has been found to be highly conserved among various mammals and is assumed to be important to the protein function. It is assumed that it is possible to be homozygous for M5, like the other known mutations, but this was not found in this sample group. No shorthair cats or longhair cats with two of the known mutations were found to have M5. All Maine coon cats with the M5 variant and one other variant were longhaired, demonstrating high to complete penetrance. No cats outside of Maine Coons were found to have the M5 variant, indicating it may be a breed specific mutation, arising after selective breeding. M5 was found in unrelated cats from different areas of the United States and Canada, showing that although it is specific to Maine Coons, it is not a private mutation. Screening of an additional 142 Maine coons did not identify any additional carriers, indicating a possible allele frequency of this mutation within the breed of about $1.4 \%$.

The variety of mutations within FGF5 in domestic animals is a remarkable example of allelic heterogeneity. Here we describe a novel variant within the FGF5 gene of Maine Coon cats causing the expression of longhair. With the addition of this variant (M5) there are five known FGF5 longhair causing variants within the Felis catus species. Adding this variant to tests offered by genetic testing facilities will 
provide additional information to breeders who use genetic results to control for certain diseases and predict the possible phenotypic outcomes for their litters.

\section{Declarations}

\section{Electronic List}

https://genome.ucsc.edu/cgi-bin/hgBlat

https://www.ncbi.nlm.nih.gov/genbank/

\section{Funding}

Funding for this study was provided by Genetic Veterinary Sciences, Inc.

\section{Conflicts of interest/Competing interest}

LGS and GDS are owners of Genetic Veterinary Sciences, Inc, DBA Paw Print Genetics which provides genetic testing on a fee-for-service basis including the CatScan genetic screen for cats. The remaining authors have no conflicts of interest to declare.

\section{Availability of data and material}

All relevant data generated in this study is included in this published article.

\section{Code availability}

Not applicable.

\section{Authors' contributions}

GS and HF-S contributed to the study conceptualization and design. Any additional sample recruitment was arranged by LGS. All experiments were performed by GS. Results were analyzed by GS and HF-S. GS produced the figures. In silico analysis was done by KM. GS wrote the first draft of the manuscript. LGS and BCB provided critical review of the manuscript. GS and BCB submitted the variant to GenBank. All authors commented on and approved the final manuscript.

\section{Ethics approval}

Not applicable.

\section{Consent to participate}

All canine samples included in this study were obtained through consent of the individual owners or were obtained from otherwise discarded DNA samples after clinical testing at Paw Print Genetics. 


\section{Consent for publication}

Not applicable.

\section{References}

1. Alhaddad H, Abdi M, Lyons LA (2021) Patterns of allele frequency differences among domestic cat breeds assessed by a 63K SNP array. PLoS One. Feb 25;16(2). [PubMed: 33630878]

2. Daverio MS, Vidal-Rioja L, Frank EN, Di Rocco F (2017) Molecular characterization of the llama FGF5 gene and identification of putative loss of function mutations. Anim Genet 48(6):716-719. [PubMed: 29024003]

3. Dierks C, Momke S, Philipp U, Distl O (2013) Allelic heterogeneity of FGF5 mutations causes the longhair phenotype in dogs. Anim Genet 44(4):425-431. [PubMed: 23384345]

4. Drögemüller C, Rüfenacht $S$, Wichert B, Leeb T (2007) Mutations within the FGF5 gene are associated with hair length in cats. Anim Genet 38(3):218-221. [PubMed: 17433015]

5. Hébert JM, Rosenquist T, Götz J, Martin GR (1994) FGF5 as a regulator of the hair growth cycle: evidence from targeted and spontaneous mutations. Cell. 23;78(6):1017-25. [PubMed: 7923352]

6. Higgins CA, Petukhova L, Harel S, Ho YY, Drill E, Shapiro L, Wajid M, Christiano AM (2014) FGF5 is a crucial regulator of hair length in humans. Proc Natl Acad Sci U S A. 22;111(29):10648-53. [PubMed: 24989505]

7. Housley DJ, Venta PJ (2006) The long and short of it: evidence that FGF5 is a major determinant of canine 'hair'-itability. Anim Genet 37(4):309-315. [PubMed: 16879338]

8. Kehler JS, David VA, Schäffer AA, Bajema K, Eizirik E, Ryugo DK, Hannah SS, O'Brien SJ, MenottiRaymond M (2007) Four independent mutations in the feline fibroblast growth factor 5 gene determine the long-haired phenotype in domestic cats. J Hered 98(6):555-566. [PubMed: 17767004]

9. Legrand R, Tiret L, Abitbol M (2014) Two recessive mutations in FGF5 are associated with the longhair phenotype in donkeys. Genet Sel Evol 25(1):65 46(. [PubMed: 25927731]

10. Yoshizawa Y, Wada K, Shimoi G, Kameyama Y, Wakabayashi Y, Fukuta K, Hashizume R (2015) A 1-bp deletion in Fgf5 causes male-dominant long hair in the Syrian hamster. Mamm Genome 26(1112):630-637. [PubMed: 26481120]

11. Yu F, Liu Z, Jiao S, Zhang X, Bai C, Zhang J, Yan S (2018) A nonsense mutation in the FGF5 gene is associated with the long-haired phenotype in domestic guinea pigs (Cavia porcellus). Anim Genet 49(3):269. [PubMed: 29603294]

12. Zhang R, Wu H, Lian Z (2019) Bioinformatics analysis of evolutionary characteristics and biochemical structure of FGF5 Gene in sheep. Gene 20:702:123-132. [PubMed: 30926307]

\section{Tables}


Table 1. List of cat breeds and number of each breed grouped by hair length. Number of mutations indicates the number of alleles identified, cats screened in silico are included in the total, but mutations M1-M4 were not evaluated. 


\begin{tabular}{|c|c|c|c|c|c|c|c|}
\hline \multicolumn{8}{|c|}{ Mutations } \\
\hline Hair Length & Breed & M1 & M2 & M3 & M4 & M5 & Total Cats Tested \\
\hline \multirow[t]{3}{*}{ Hairless } & Bambino & - & - & - & - & - & 1 \\
\hline & Peterbald & - & - & - & - & - & 1 \\
\hline & Sphynx & - & - & - & 2 & - & 11 \\
\hline \multirow[t]{7}{*}{ Longhair } & Birman & - & - & - & 2 & - & 1 \\
\hline & British Longhair & - & 1 & - & 9 & - & 5 \\
\hline & Maine Coon & 1 & - & 96 & 192 & 4 & 146 \\
\hline & Norwegian Forest Cat & - & 3 & - & 1 & - & 6 \\
\hline & Persian & - & - & 1 & 11 & - & 14 \\
\hline & Ragdoll & 9 & - & 11 & 26 & - & 23 \\
\hline & Siberian & - & 2 & - & 10 & - & 6 \\
\hline \multirow[t]{15}{*}{ Shorthair } & Abyssinian & - & - & - & - & - & 3 \\
\hline & British Shorthair & - & 1 & - & - & - & 5 \\
\hline & Burmese & - & - & - & - & - & 2 \\
\hline & Chausie & - & - & - & 2 & - & 5 \\
\hline & Cornish Rex & - & - & - & 1 & - & 5 \\
\hline & Egyptian Mau & - & - & - & - & - & 2 \\
\hline & Exotic Shorthair & - & - & - & 2 & - & 3 \\
\hline & Highlander & - & - & - & - & - & 1 \\
\hline & Jungle Cat & - & - & - & - & - & 1 \\
\hline & Oriental & - & - & - & 1 & - & 1 \\
\hline & Oriental Shorthair & - & - & - & - & - & 2 \\
\hline & Russian Blue & - & - & - & - & - & 1 \\
\hline & Savannah & - & - & - & 1 & - & 4 \\
\hline & Thai & - & - & - & 1 & - & 1 \\
\hline & Tonkinese & - & - & - & - & - & 5 \\
\hline \multirow[t]{2}{*}{ Short/Long hair } & Bengal & - & - & - & 1 & - & 6 \\
\hline & Devon Rex & - & - & 5 & 6 & - & 14 \\
\hline
\end{tabular}




\begin{tabular}{|c|c|c|c|c|c|c|c|}
\hline & Domestic Cat & - & - & - & 1 & - & 45 \\
\hline & Pixie-Bob & - & - & - & 1 & - & 1 \\
\hline & Scottish Fold & - & 1 & - & 11 & - & 10 \\
\hline & Scottish Straight & - & - & - & 7 & - & 8 \\
\hline & Selkirk Rex & - & - & - & 3 & - & 2 \\
\hline & Siamese & - & - & - & 1 & - & 4 \\
\hline Total & & 10 & 8 & 113 & 292 & 4 & 345 \\
\hline
\end{tabular}

Table 2. Primer sequences used to identify the M5 variant

\begin{tabular}{|lll|}
\hline Primer Name & Forward & Reverse \\
\hline FGF5_1 & 5'-AATGAACACTTGACTGCTAGGC-3' & 5'-AGTCCTCTAAGCAAATTGTCCC-3' \\
\hline FGF5_6 & 5'-AGTTTCCTGAGTTCAAGCCCC-3' & 5'-AGTGTAGCTGCAGGTGTATCC-3' \\
\hline FGF5_5 & 5'-GAGGAAGTTTTCCGTCAGTGG-3' & 5'-AAGTGGGTAGAGATGTGCTGG-3' \\
\hline FGF5_9 & 5'-TTCCATCTGCAGATCTACCCG-3' & 5'-TAGATGCACCTTCACCCAACC-3' \\
\hline
\end{tabular}

\section{Figures}

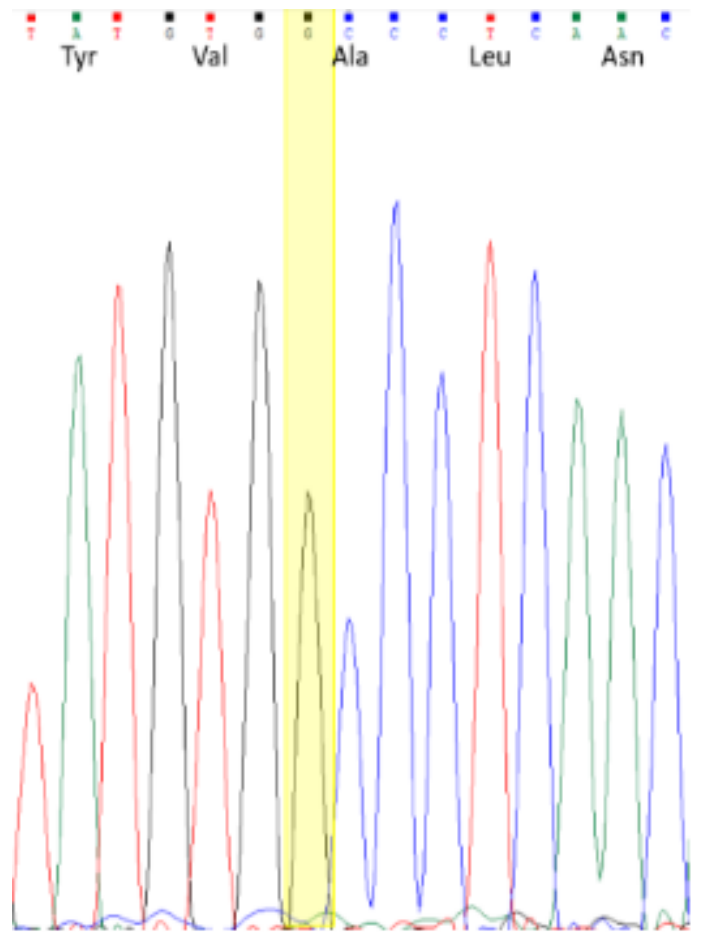

Control Cat

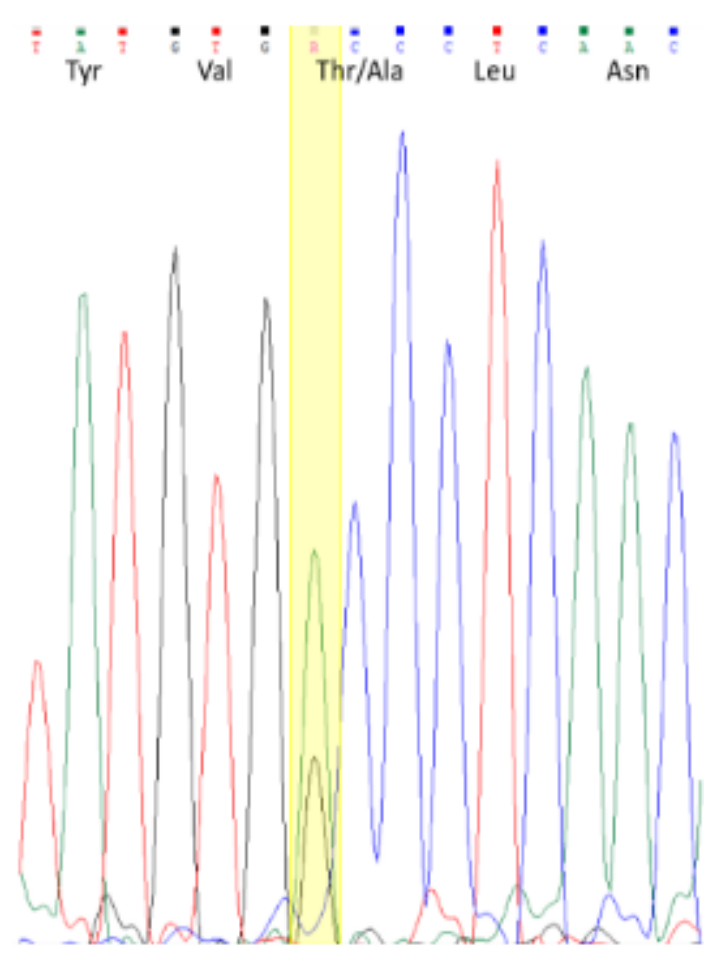

Test Cat

Figure 1 
Sanger sequencing chromatograms shown for unrelated control and test cats showing variant wildtype position and variant heterozygous position, wildype is homozygous for $G / G$ and the test cat is heterozygous for $A / G$

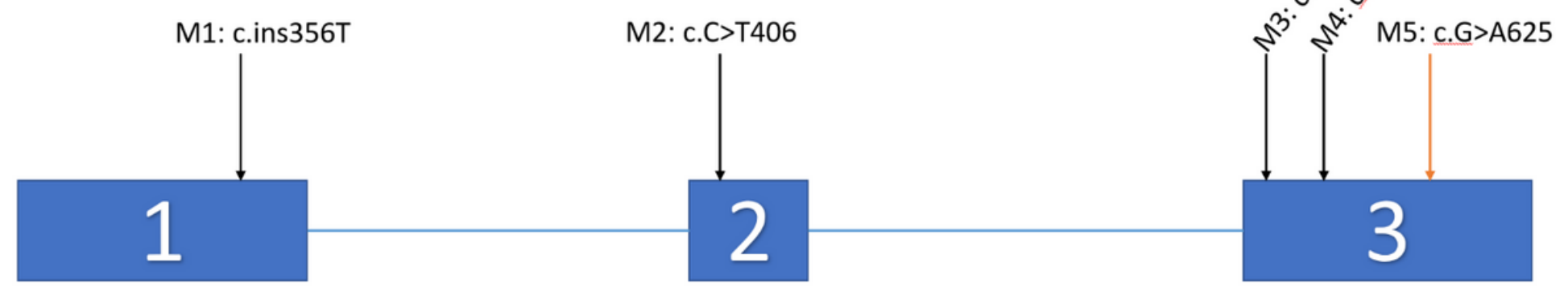

Figure 2

Map of FGF5 showing positions of known mutations and new Mutation 5 (M5), each exon is represented by a number (1-3).

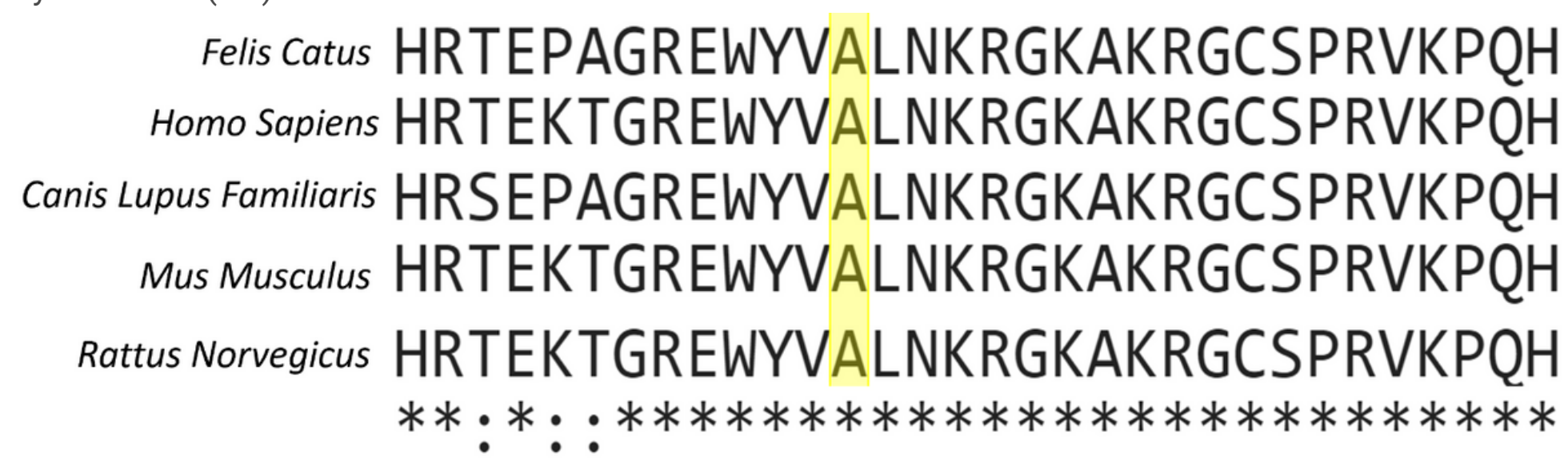

Figure 3

Alignment of amino acid sequence showing A193 is highly conserved among species 


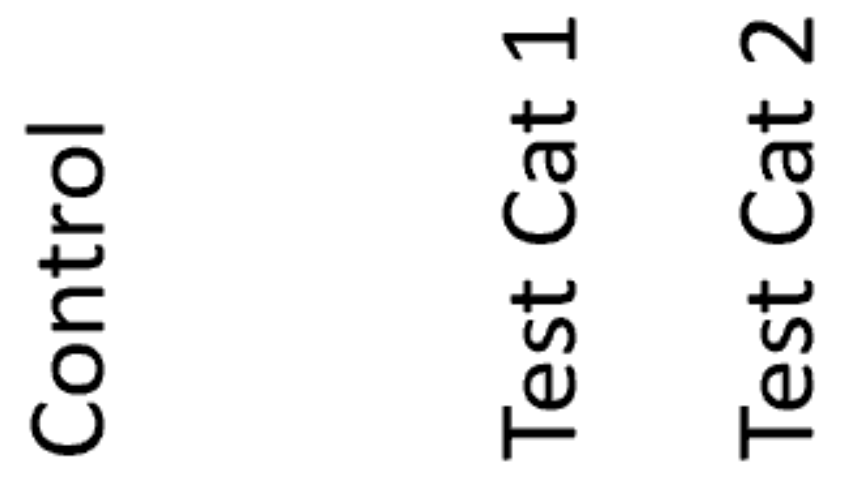

\section{$600 \mathrm{bp}$ $400 \mathrm{bp}$ $350 \mathrm{bp}$}
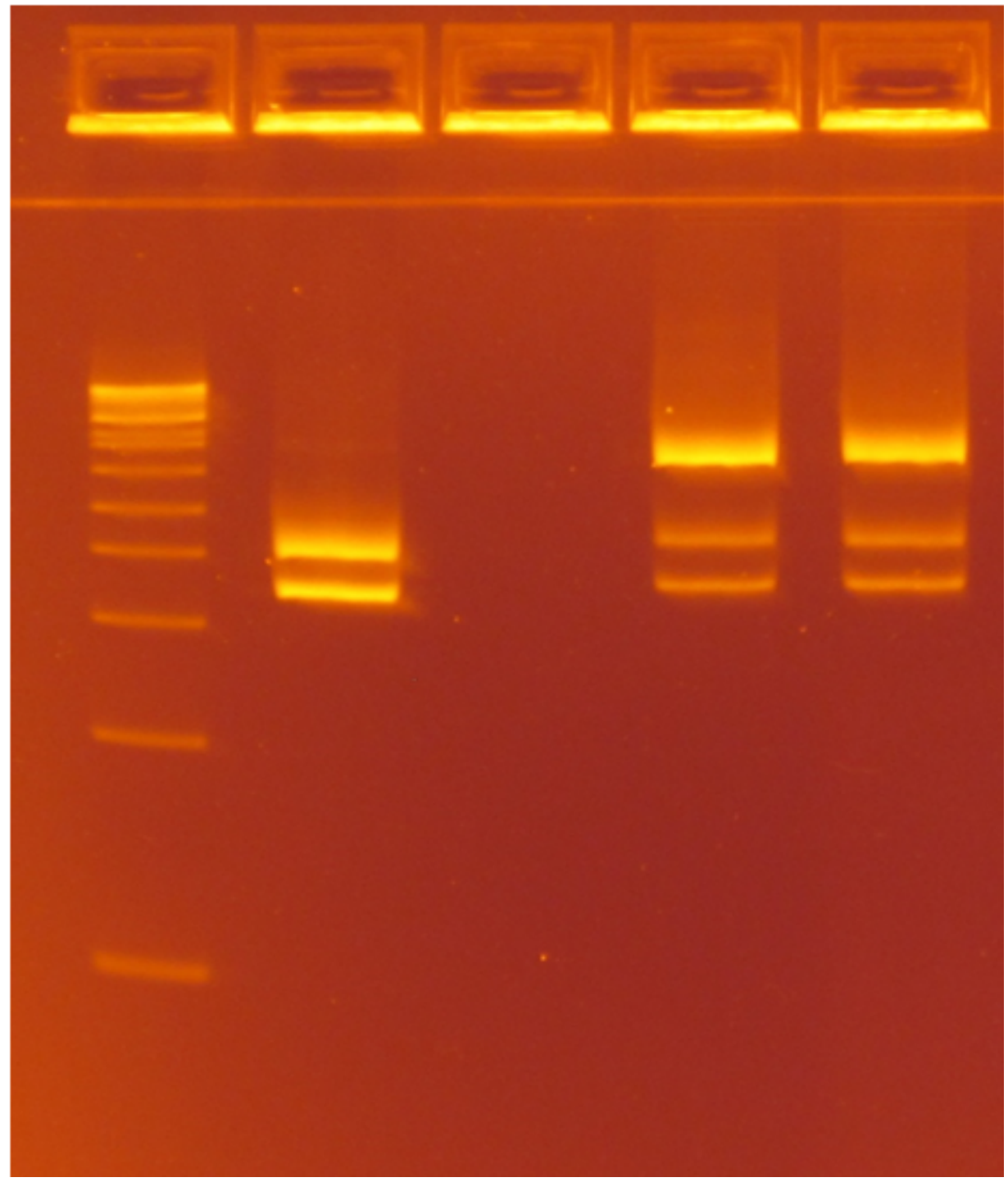

Figure 4

PCR screening assay showing banding patterns after gel electrophoresis of wildtype and heterozygous Mutation 5 cats 
A

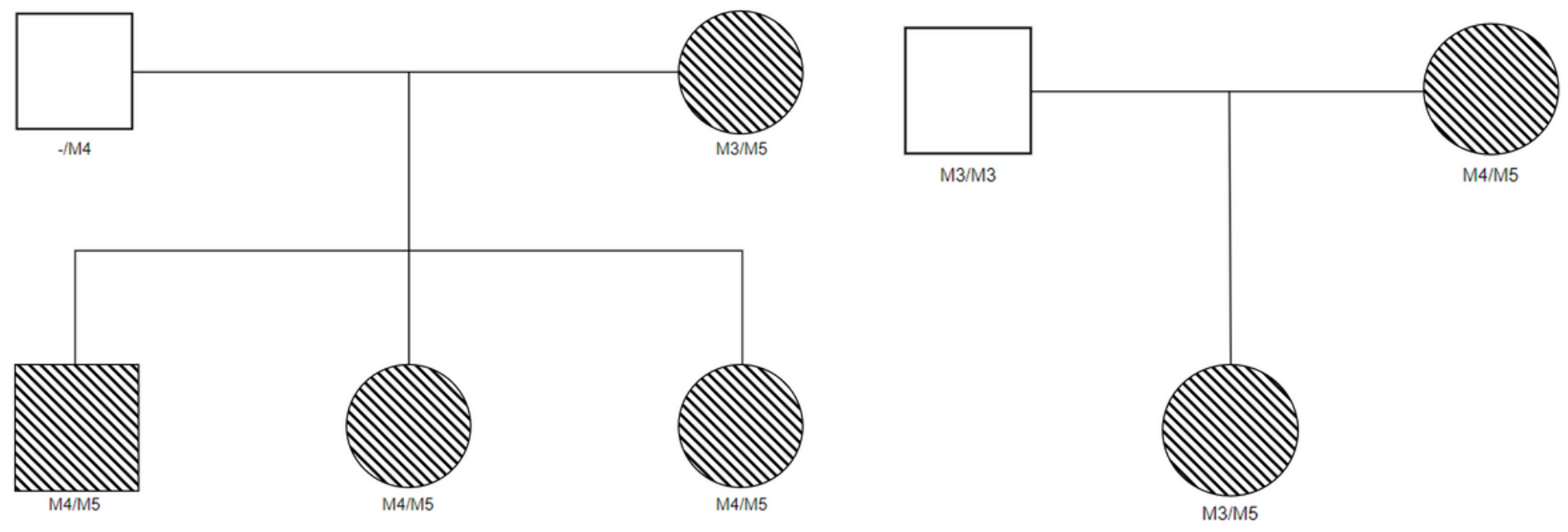

Figure 5

Pedigrees showing inheritance of the M5 variant. Circles are female, squares are male. Hatch pattern indicates individuals compound heterozygous for M5. A and B represent two unrelated pedigrees. Genotypes in the father shown in A are inferred. 